\title{
REVIEWER ACKNOWLEDGMENT 2014
}

Reviewing manuscripts is arduous and time-consuming, yet it is the cornerstone of the peer-review system that advances knowledge. Genetics in Medicine thanks the following reviewers and editors who were kind enough to give us their time in 2014.

Louise Acheson

Fowzan Alkuraya

Dawn Allain

Melissa Austin

Karen Avraham

Nazneen Aziz

Janice Bach

Don Bailey

Michela Barbaro

Daniel Barocas

David Basel

Genevieve Baujat

Samantha Baxter

Lora Bean

Michael Beck

Cecelia Bellcross

Peter Benn

Robin Bennett

Jonathan Berg

Ben Berkman

Carmelo Bernabeu

Barbara Bernhardt

Alison Bertuch

Laura Beskow

Robert Best

Katherine Bianco

Barbara Biesecker

Leslie Biesecker

Laurence Bindoff

Cinnamon Bloss

Stefania Boccia

Nadege Bondurand

Vence Bonham

Jessica Booker

Guntram Borck

Jeffrey Botkin

Deborah Bowen

Angela Bradbury

Kyle Brothers

Damien Bruno

The-Hung Bui

Wylie Burke

Barbara Burton

Peter Byers

R. Jean Cadigan

John Carey

Stephen Cederbaum
Gary Chadwick

Subhashini Chandrasekharan

WeiChiao Chang

Audrey Chapman

Alka Chaubey

Chin-Fu Chen

Haoyan Chen

Edith Cheng

Mark Chestnutt

David Chitayat

Bradford Coffee

Michael Cohen

Celeste Condit

Laura Conlin

Harriet Corvol

Catherine Cottrell

Fergus Couch

Howard Cuckle

Garry Cutting

Michael Datto

Mariska den Heijer

Shweta Dhar

Harry Dietz

Megan Doerr

Rajkumar Dorajoo

Michael Dorschner

W. Dotson

Kirsten Douma

Sophie Dupuis-Girod

Jon Emery

Gregory Enns

Barbara Evans

Gareth Evans

James Evans

Jessica Everett

Declan Fahy

Antonio Farina

Lisa Feuchtbaum

Ridgely Fisk Green

James Ford

Jan Friedman

Stephanie Fullerton

Birgit Funke

Merce Garcia-Barcelo

Elizabeth Gillanders

Aaron Goldenberg

Paula Goldenberg
Dorothy Grange

Wayne Grody

Scott Grosse

Muge Gucsavas Calikoglu

Susanne Haga

Jiali Han

Paul Harmatz

Madhuri Hegde

Gail Henderson

Lindsay Henderson

Edward Highsmith

Jennelle Hodge

Ingrid Holm

Yuling Hong

Robert Hopkin

Claude Houdayer

Christopher Howard Wade

Roeland Huijgen

Sara Hull

Steve Humphries

Elizabeth Jameson

Kory Jasperson

Brian Jensen

Steven Joffe

U. Kalyoncu

John Kastelein

Lorraine Katz

David Kaufman

Hutton Kearney

Y. Kee

Martin Kharrazi

Muin Khoury

Nancy King

Russell Kirby

Salman Kirmani

Robert Klein

Bartha Knoppers

Josh Knowles

Barbara Koenig

Katherine Kolor

Bruce Korf

Ruth Kornreich

Andrzej Kosinski

Ian Krantz

Donna Krasnewich

Allen Lamb

Andrew Latchford
Alexander Lazar

Brandie Leach

David Ledbetter

Kristy Lee

Nicholas Lench

Debra Leonard

Kathleen Leppig

Megan Lewis

Ute Lichter-Konecki

Chuck Lidz

Feng-Chang Lin

Noralane Lindor

Julian Little

Xiaoming Liu

Bart Loeys

Nicola Longo

Troy Lund

Henry Lynch

Julie Lynch

Patrick Lynch

Anne Madeo

Everett Magann

MR Mailick

Gary Marchant

Pamela Marcus

Ali Marian

Joan Marini

Barbara Marriage

Jessica McAlpine

Donna McDonald-McGinn

Amy McGuire

Howard McLeod

Andrew McNaught

David Miller

Anna Mitchell

Yuka Mizusawa

Jessica Moline

Zahra Montazeri

Eva Morava

Tomi Mori

Ian Morison

John Mulvihill

Michael Murray

Adele Murrell

Katherine Nathanson

Marvin Natowicz

Mary Norton 


Robert Nussbaum
Godfrey Oakley Jr
Barbara O’Brien
Julianne O’Daniel
Suzanne O’Neill
Kelly Ormond
Brian O'Roak
Tuya Pal
Glenn Palomaki
Nora Pashayan
Gregory Pastores
Andrea Patenaude
Markus Perola
June Peters
Susan Peterson
Kathryn Phillips
Antonio Pisani
Sharon Plon
Rodney Pollitt
Lori Potocki
Beth Potter
Cynthia Powell
Siddharth Prakash
Anya Prince
Thomas Prior
Michele Puryear
Susan Puumala
Reed Pyeritz
Ming Qi
Matthew Rabinowitz

Nazneen Rahman
Arti Rai
David Ransohoff
Katherine Rauen
Vincent Raymond
Heidi Rehm
Marian Reiff
Rosemary Reiss
Pam Renwick
Aurelio Reyes
Vincent Riccardi
Greg Rice
Piero Rinaldo
Chris Rini
J. Scott Roberts
Mark Robson
Myra Roche
Jill Rosenfeld
Eric Rosenthal
Mark Rothstein
Heiko Runz
Carlo Sabba
Srirangan Sampath
Saskia Sanderson
Jonathan Sanford Berg
Avni Santani
Glen Satten
Gerald Schaefer
Stephen Scherer
Maren Scheuner

John Schmitz
Iris Schrijver
Marc Schwartz
Daryl Scott
Joan Scott
Praveen Sethupathy
Richard Sharp
Jay Shendure
Marwan Shinawi
Brian Shirts
Ellen Sidransky
Sandra Sirrs
Debra Skinner
Brian Skotko
Cécile Skrzynia
Zsofia Stadler
James Stavropoulos
Robert Steiner
Olivier Sterkers
Harvey Stern
Elena Stoffel
V. Reid Sutton
Holly Tabor
Ozlem Tastan Bishop
Mustafa Tekin
Kenneth Tercyak
Steven Teutsch
John Thorson
S. Tierney
Marc Tischkowitz

Ian Tomlinson

Karen Tsuchiya

Clare Turnbull

Keiko Ueda

David Veenstra

Karl Voelkerding

Neeta Vora

Peggy Walker

Tomas Walsh

Catharine Wang

Michael Watson

Gerald Watts

Stephanie Wechsler

Karen Weck

Robert Weintraub

Rosanna Weksberg

Corrine Welt

Catherine Wicklund

Benjamin Wilfond

Andrew Wilkie

Janet Williams

Marc Williams

Susan Wolf

Ellen Wright Clayton

Yaping Yang

Patricia Zartman Page

Elvira Zilliacus

Andrew Zinn

If you reviewed for us in 2014 and are not on this list, please contact the managing editor, Jan Higgins, at gim@acmg.net and we will include you in our next issue. 